\title{
Research Paper: Kinematics of Lower Extremity During Forward and Backward Walking on Different Gradients
}

\author{
Sasan Naderi ${ }^{\text {* }}$, Fariborz Mohammadipour ${ }^{1} \mathbb{D}$, Mohammad Reza Amir Seyfaddini $^{1}$
}

1. Department of Biomechanics, School of Physical Education and Sport Sciences, Shahid Bahonar University of Kerman, Kerman, Iran.

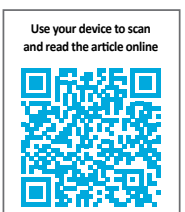

Citation: Naderi S, Mohammadipour F, Amir Seyfaddini MR. Kinematics of Lower Extremity During Forward and Backward Walking on Different Gradients. Physical Treatments. 2017; 7(2):71-78. http://dx.doi.org/10.32598/ptj.7.2.71

Article info:

Received: 16 Dec 2016

Accepted: 05 Apr 2017

Keywords:

Forward walking, Backward walking, Lower extremity kinematics, Sloped surfaces

\begin{abstract}
A B S T RA C T
Purpose: Forward Walking (FW) and Backward Walking (BW) on different gradients of the treadmill is a common exercise for lower extremity rehabilitation. However, limited studies are found about the three-dimensional analysis of lower extremity and status of the knee joint during FW and BW on different gradients. Therefore, this study aimed to investigate the lower extremity joints kinematics during FW and BW on incline and decline surfaces.
\end{abstract}

Methods: The current research has a quasi-experimental design. Sixteen healthy males with the mean (SD) age of 22.4(2.5) years, volunteered to participate in this study. The subjects' FW and BW with their preferred speed on a treadmill at four gradients $(-7.5 \%, 0 \%,+5 \%$ and $+10 \%)$, were analyzed by using motion capture system. All data were analyzed using paired sample $t$ test $(\mathrm{P}<0.05)$.

Results: Significant differences were seen between FW and BW in most angular variables in sagittal plane. However, there were no significant differences between FW and BW in most parameters of knee angle in frontal plane.

Conclusion: Based on the results, with increase in $\mathrm{FW}$ inclination and decrease in BW inclination, the knee varus angle in frontal plane reduces. Therefore, $\mathrm{FW}$ on the upslope surfaces or BW on downslope surfaces, probably is a suitable solution to reduce the loads exerted on medial compartment of the knee.

\section{Introduction}

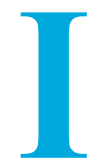

$n$ recent years, closed chain exercises have received extensive attention by physiotherapist and sports coaches and used in rehabilitation programs for lower extremity injuries. These exercises are safer, more effective, and more functional than the open chain exercises $[1,2]$. Forward Walking (FW) and Backward Walking (BW) are the most commonly used closed chain exercises. However, researchers have reported that BW may have other benefits besides FW exercises. These benefits are increased muscle activity, increased oxygen consumption, metabolic and cardio-respiratory

\footnotetext{
* Corresponding Author:

Sasan Naderi, MSC.

Address: Department of Biomechanics, School of Physical Education and Sport Science, Shahid Bahonar University of Kerman, Kerman, Iran. Phone: +98 (937) 0300595

E-mail: sasan_naderi1369@yahoo.com
} 
response, and reduction of pressure on the joints [2, 3]. Backward walking and jogging exercises are also used for muscle building, improving sport performance and promoting balance $[4,5]$.

On the other hand, FW on sloping surfaces is used with the objectives of training and rehabilitation. Walking on sloping surfaces increases activities of quadriceps, hamstrings, gastrocnemius, and soleus muscles. This results in increased metabolism, fat burning, as well as increased muscle strength of the lower limbs [6,7]. This kind of walking is recommended and prescribed for fitness to the elderly and people with obesity, for knee joint rehabilitation, also to patients who underwent complete knee replacement, and finally to people with knee varus in order to reduce pressure to the medial compartment of the knee joint [8-10]. Walking on upward sloping surfaces is recommended because it decreases torque arm of Ground Reaction Force (GRF) on the center of the knee joint. In a natural state, forces applied to the shin tend to create flexion-extension and adduction-abduction movements in the knee joint. Research evidence has shown that while walking, GRF to the center of the joint passes through the medial surface of the knee joint. Applying the force around the knee joint center produces a torque which tends to put the knee in adduction position [11].

The created torque has an important contribution in total pressure on the knee joint, influenced by the size of the GRF, torque arm of the reaction force around the center of the knee joint (which is defined as the vertical distance between the line of action of this force and center of knee rotation), as well as mass and acceleration of the various parts of the lower limb. Greater torque arm results in higher pressure load over the medial part of the knee. It is to be noted that knee joint position on the frontal plane plays an important role in the joint health and increased knee adduction has direct relationship with the progression of articular diseases and the medial compartment osteoarthritis [12, 13]. Researchers reported that in healthy subjects, the maximum reaction force of the knee joint is about 3.2 times the weight of the body which almost $70 \%$ of it passes through the medial compartment of the knee joint [14].

It seems necessary to find a solution to reduce the load applied to the medial compartment of the knee joint of people with articular diseases and other patients suffering from increased load over this part of the knee joint. Although, little research has been done in this field, the results indicate that in FW, increased incline of the walking path leads to decreased knee adduction and by decreasing the incline, this angle increases $[9,10]$. How- ever, it has not been determined yet that how this angle changes in BW on different slopes. Therefore, the primary purpose of the research was comparing the angle of the knee joint in the frontal plane and the secondary objective is to compare the angles of lower limb joints on the sagittal plane between FW and BW while taking steps on the different inclines.

\section{Materials and Methods}

The current study has quasi-experimental design. The statistical population comprised all male students of Shahid Bahonar University of Kerman. Based on the relevant studies, the sample size was estimated to be at least 15 people to satisfy the statistical power of 0.75 at a significant level of 0.05 [15]. Of the statistical population, 16 healthy male subjects (with a mean [SD] age of 22.4[2.35] years, height of 175.5 [6.00] cm, weight of $68.6[8.47] \mathrm{kg}$, the distance between two femur medial epicondyles of $0.76[0.51] \mathrm{cm}$, self-select FW speed of $4.88[0.41] \mathrm{km} / \mathrm{h}$, and the self-select BW speed of $92.9[0.54] \mathrm{k} / \mathrm{h}$ ) were chosen purposefully. They had regular physical activity (at least three sessions per week). The subjects were also examined and evaluated with respect to the distance between the two medial epicondyles of femur and two medial malleolus using caliper (Mitutoyo models; Vernier and Long Jaw), their foot planar position using the mirror box, and other criteria. Finally, the individuals were selected who lacked any injuries or disorders in their lower limbs. This study was approved by the Ethics Committee of Shahid Bahonar University of Kerman and all subjects signed the informed consent forms for participation in the research.

After the demographic assessment of the subjects, they were asked to walk 10-m at their own paces, three times forward and three times backward with bare feet and the their walking durations were recorded with a stopwatch. After completing three walks, the average speed was used as the individual's own speed in order to adjust treadmill speed (TUNTURI J880, Almere, NL) [16].

Eleven passive reflective markers were installed on the lower limbs of the subjects based on the plug-in-gait model. The position of these markers were on greater trochanter, lateral femur epicondyle, lateral malleolus, head of the first tarsal bone, the midpoint on the line that connects the anterior superior iliac spine to patella, tibial tubercle, anterior and middle part of the shin over the distal part of the tibia, right and left foot calcaneus and big toe bones (Figure 1) [17-19]. Markers were fixed on subjects' bodies using double-sided adhesive and rubber. Markers positions were recorded using the 3D motion 
analysis system with 6 Optoelectronic Cameras (Rapture H Motion Analysis System, Santa Rosa, CA) with a frequency of $120 \mathrm{~Hz}$.

First, each subject stood in front of the camera in anatomical position. The position of the markers of each subject was recorded for 5 seconds in the standing situation and then enough time was given to the subjects to feel comfortable with walking on the treadmill. Subjects, then, walked with self-selected speed and in random order on the inclines of $0,+5,+10$, and -7.5 percentages $(0,+9,+18$ and 13.5 degrees, respectively). After walking for $2 \mathrm{~min}$ on each inclines, $20 \mathrm{~s}$ recording took place [18-20]. In order to prevent fatigue, after each test, the subject could rest for $1 \mathrm{~min}$. After completing the FW tests, each subject was given 5 min rest and then the BW tests were conducted.

The obtained data were analyzed by the system of motion analysis with CORTEX 2.5 (Motion Analysis Corporation, Santa Rosa, CA). This application can create biomechanical motion model and calculate joints angles. To reduce the noise caused by the motion of the markers and smooth the data, butterworth lowpass filter with the cut-off frequency of $6 \mathrm{~Hz}$ was used [21]. In order to reduce data, the recorded video of five consecutive steps was extracted.

Walking events and temporal-spatial variables were also calculated using speed-based treadmill algorithm and MATLAB (MathWorks Inc., Natick, MA) [22]. Using this algorithm and heel marker coordinates, heel contact (toe touching in BW) is determined when the speed component in the X direction (along the subject's walking path) switches from positive values to negative ones. In addition, when this component switches from negative values to positive ones (heel-off in BW), it is considered as heel-off. Eventually, parameters of hip angle, knee angle (on sagittal and frontal planes), ankle angle at heel contact positions (toe touching in BW), mid-stance and toe-off (heel-off in BW) were calculated (the thigh joint angle was absolute and the other angles were relative).

Statistical analysis of data was carried out using SPSS 22 (SPSS Inc., Chicago, IL) at 0.05 level of significance. After determining data normalization through the Shapiro-Wilk test, analysis of variance with repeated measurements of the $2 \times 4$ composite design was used to compare angular parameters of $\mathrm{FW}$ with $\mathrm{BW}$ when walking on different inclines. After the significance of the differences was established, $t$ test was used to determine the significant points.

\section{Results}

Table 1 presents characteristics of subjects including age, height, mass, and velocity of self-selected FW and BW. Table 2 presents temporal-spatial parameters. The results did not show the significant differences between FW and BW and also between inclines of $-7.5,+5$, and +10 percentages and 0 incline.

Table 3 presents values related to the angular parameters of the hip, knee, and ankle joints on the sagittal plane. The results of t-correlation test showed that there is a significant difference between FW and BW in most angular parameters on the sagittal plane (Table 3, Figure 2).

Table 4 presents knee joint angular parameter on the frontal plane. The results generally showed that with increasing incline in $\mathrm{FW}$, the knee angle values reduce on the frontal plane and in BW, the knee angle values on the frontal plane increase. However, there were no significant differences between FW and BW in most of the examined parameters (Table 4, Figure 2).

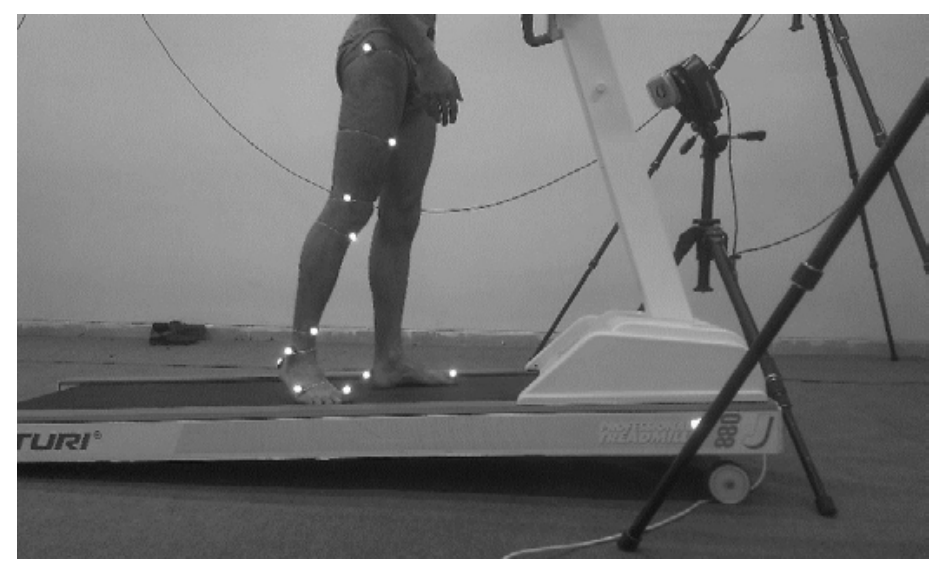

Figure 1. Position of 11 passive reflection markers 

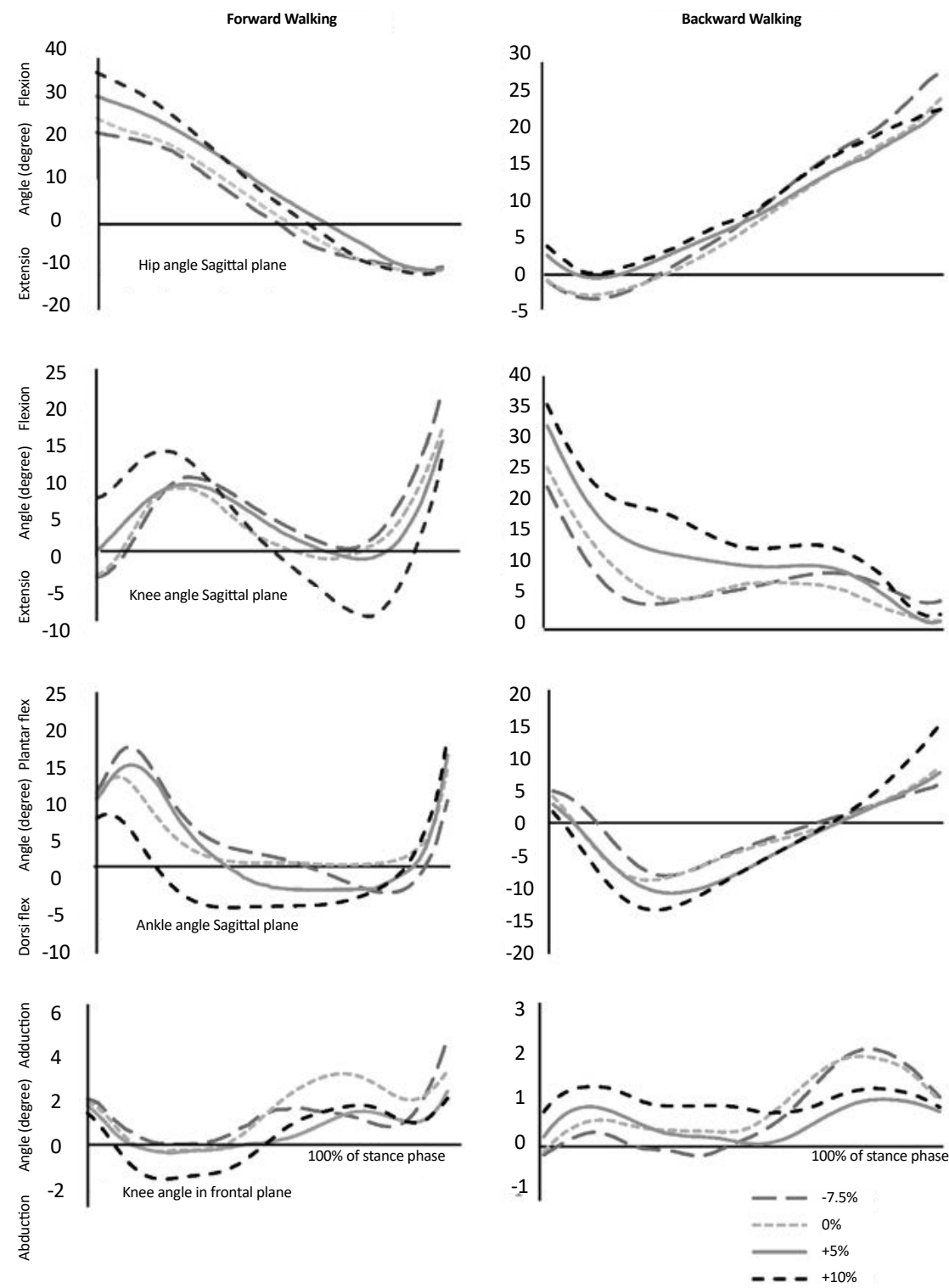

PHYSICAL TREA M MENTS

Figure 2. Kinematic diagrams of hip, knee and ankle angles when walking forward and backwards on four inclines (the mean joint angles of all subjects)

\section{Discussion}

The purpose of this study was to compare lower limb kinematics during FW and BW on different inclines. The results of this study showed that by increasing the incline of walking, angle of flexion in the hip and knee joints and dorsiflexion angle of the ankle joint at heel contact moment and mid-stance increases. Also, at the toe-off moment, increase in hip and knee joints extension and ankle plantar flexion were observed. The results on BW also indicate a slight increase in hip joint flexion at the moments of toe contact and mid-stance and flexion reduction at the moment of toe-off with increased incline of walking. Knee joint in BW bends more with increasing walking incline and its flexion increased on toe touching and mid-stance position. But at toe-off moment, no significant change was seen. Also with regard to ankle joint, with increasing inclines, plantar ankle flexion reduces at the moment the toe touches the ground; at the moment of mid-stance the ankle is almost in its natural state, and at the moment the heel is detached, plantar flexion increases.

In $\mathrm{FW}$, with an incline increase from $-7.5 \%$ to $+10 \%$, the mechanism of taking steps is as follows: hip joint has flexion in continuation of the legs forward sway in swing phase at the moment of heel contact, and when walking 
Table 1. Demographic characteristics of the subjects

\begin{tabular}{cc} 
Index & Mean \pm SD \\
\hline Age $(\mathrm{y})$ & $22.4 \pm 2.57$ \\
Height $(\mathrm{cm})$ & $175.5 \pm 6.00$ \\
Mass $(\mathrm{kg})$ & $68.6 \pm 8.47$ \\
Self-selected FW $(\mathrm{km} / \mathrm{h})$ & $4.88 \pm 0.41$ \\
Self-selected BW $(\mathrm{km} / \mathrm{h})$ & $3.92 \pm 0.54$ \\
\hline & $\underline{\text { PHYSICAL TREATMENTS }}$
\end{tabular}

incline increases, the flexion also increases (from 21.6 degrees to 35.18 degrees) to overcome this challenge. At this moment, knee joint is almost extended completely at different inclines (from 2.6 degrees of hyperextension to 8.06 degrees of flexion) and the ankle joint also has 8.62 degrees of plantar flexion that reduces to 63.5 degrees with an incline increase. In the next phase of the stance, the amount of flexion in the hip joint reduces and turns into hypertension at the moment of toe-off (approximately 10 degrees of hypertension in all inclines). With an approximate fixed hip joint angle, knee joint changes it position to adapt to different inclines and with an incline increase, its flexion decreases by 20.44 degrees (from 22.75 degrees to 2.31 degrees) and plantar ankle flexion also increases (from 7.67 to 10.15 degrees).

However in BW, contrary to FW, at the moment of toe touch contact, the hip joint does not change much with an incline increase (from 0.81 degrees of hypertension to 3.92 degrees of flexion) but knee joint flexion degree increases from 22.40 to 35.29 degrees and the ankle joint plantar flexion decreases slightly (from 4.95 to 1.67 degrees). At the end of the stance phase and at the moment of heel-off, the knee joint angle is approximately constant with increasing incline (from 4.45 degrees of flexion to 2.31) and hip joint angles change from 27.62 degrees to 22.56 degrees, and especially the ankle joint plantar flexion from 5.98 degrees to 15.18 degrees.
Regarding the angle of the knee on the frontal plane, the results of this study showed that BW generally affects the knee angle to a lesser degree in the frontal plane, compared to FW. During BW, motion ranges of hip, knee, and ankle joints reduce and the length of the step also decreases [23]. Furthermore, vertical Ground Reaction Force (GRF) is less in BW than FW. Vertical GRF is a factor affecting the knee angle in the frontal plane in such a way that during FW, the highest knee adduction rate is when the vertical GRF reaches its maximum amount when bearing weight and heel-off moment. In FW and BW, vertical GRF has two main peak forces. In $\mathrm{BW}$, the primary peak force is created by tolerating body weight and maintaining stability after toe touching the ground. It is almost equal to the initial FW peak force. But the secondary peak force, which is created by the heel push-off in BW, is less than that of FW [24].

These factors absorb stress to the joints, therefore angle of the knee on the frontal plane has less adduction during $\mathrm{BW}$ than in FW that leads to reduction in the torque of the GRF around the knee joint center. Adduction torque, which is an effective and important parameter in studying the walking of the people with genu varum, has a direct relationship with ground force and the torque arm of this force during the stance phase of walking. Tetsworth and Paley (1994) and Brouwer (2007) showed in their research that $4 \%$ to $6 \%$ increase in knee adduction angle leads to $20 \%$ pressure increase to the medial surface of

Table 2. Spatial-temporal variables presented as mean \pm SD during walking forward and backward on different inclines

\begin{tabular}{|c|c|c|c|c|c|c|c|c|c|}
\hline & \multicolumn{9}{|c|}{ Walking Incline } \\
\hline & & $-7.5 \%$ & $\mathbf{P}$ & $0 \%$ & $\mathbf{P}$ & $+5 \%$ & $\mathbf{P}$ & $+10 \%$ & $\mathbf{P}$ \\
\hline \multirow[t]{2}{*}{ Stance duration, s } & FW & $0.02 \pm 0.54$ & \multirow[t]{2}{*}{0.809} & $0.02 \pm 0.54$ & \multirow[t]{2}{*}{0.724} & $0.02 \pm 0.56$ & \multirow[t]{2}{*}{0.602} & $0.02 \pm 0.57$ & \multirow[t]{2}{*}{0.296} \\
\hline & BW & $0.04 \pm 0.53$ & & $0.03 \pm 0.53$ & & $0.03 \pm 0.53$ & & 0.040 .52 & \\
\hline \multirow[t]{2}{*}{ Duration of taking one step, $s$} & FW & $0.04 \pm 0.95$ & \multirow[t]{2}{*}{0.241} & $0.04 \pm 0.95$ & \multirow[t]{2}{*}{0.280} & $0.04 \pm 0.97$ & \multirow[t]{2}{*}{0.112} & $0.03 \pm 0.98$ & \multirow[t]{2}{*}{0.064} \\
\hline & BW & $0.07 \pm 0.89$ & & $0.06 \pm 0.90$ & & $0.05 \pm 0.88$ & & $0.06 \pm 0.87$ & \\
\hline \multirow{2}{*}{ Step length $(\mathrm{cm})$} & $\mathrm{FW}$ & $10.52 \pm 122.24$ & \multirow{2}{*}{0.078} & $9.20 \pm 124.42$ & \multirow{2}{*}{0.053} & $10.24 \pm 128.08$ & \multirow{2}{*}{0.065} & $10.30 \pm 129.94$ & \multirow{2}{*}{0.106} \\
\hline & BW & $11.78 \pm 101.26$ & & $10.66 \pm 104.58$ & & $8.22 \pm 106.84$ & & $7.62 \pm 107.12$ & \\
\hline
\end{tabular}


Table 3. Angles of hip, knee and ankle in sagittal plane presented as mean $\pm S D$ while walking back and forth on different inclines

\begin{tabular}{|c|c|c|c|c|c|c|c|c|}
\hline Joint & Incline & FW/BW & $\begin{array}{l}\text { Heel Contact Moment in } \\
\text { FW and Toe Contact in BW }\end{array}$ & $\mathbf{P}$ & Mid-Stance & $\mathbf{P}$ & $\begin{array}{l}\text { Heel-off Moment in FW } \\
\text { and Toe-off in BW }\end{array}$ & $\mathbf{P}$ \\
\hline \multirow{8}{*}{$\begin{array}{l}\text { Hip } \\
\text { (angle) }\end{array}$} & \multirow{2}{*}{$-7.5 \%$} & FW & $21.16 \pm 4.09$ & \multirow{2}{*}{$0.001^{*}$} & $5.28 \pm 4.28$ & \multirow{2}{*}{$0.001^{*}$} & $-10.23 \pm 4.50$ & \multirow{2}{*}{$0.001^{*}$} \\
\hline & & BW & $-0.81 \pm 3.77$ & & $10.56 \pm 3.94$ & & $27.62 \pm 4.16$ & \\
\hline & \multirow{2}{*}{$0 \%$} & FW & $24.49 \pm 4.29$ & \multirow{2}{*}{$0.001^{*}$} & $4.55 \pm 1.62$ & \multirow{2}{*}{$0.001^{*}$} & $-10.99 \pm 4.52$ & \multirow{2}{*}{$0.001^{*}$} \\
\hline & & BW & $-0.73 \pm 5.17$ & & $9.22 \pm 5.07$ & & $24.00 \pm 2.82$ & \\
\hline & \multirow{2}{*}{$+5 \%$} & FW & $29.59 \pm 4.92$ & \multirow{2}{*}{$0.001^{*}$} & $6.80 \pm 5.00$ & \multirow{2}{*}{${ }^{*} 0.001$} & $-10.75 \pm 4.52$ & \multirow{2}{*}{$0.001^{*}$} \\
\hline & & BW & $2.67 \pm 4.96$ & & $12.06 \pm 5.13$ & & $22.55 \pm 3.48$ & \\
\hline & \multirow{2}{*}{$+10 \%$} & FW & $35.18 \pm 5.34$ & \multirow{2}{*}{$0.001 *$} & $8.15 \pm 4.90$ & \multirow{2}{*}{${ }^{*} 0.001$} & $-11.35 \pm 4.05$ & \multirow{2}{*}{$0.001^{*}$} \\
\hline & & BW & $3.92 \pm 4.07$ & & $13.50 \pm 4.11$ & & $22.56 \pm 3.08$ & \\
\hline \multirow{8}{*}{$\begin{array}{l}\text { Knee } \\
\text { (angle) }\end{array}$} & \multirow{2}{*}{$-7.5 \%$} & FW & $-2.69 \pm 2.78$ & \multirow{2}{*}{$0.001^{*}$} & $8.89 \pm 4.08$ & \multirow{2}{*}{$* 0.001$} & $22.75 \pm 3.28$ & \multirow{2}{*}{$0.001^{*}$} \\
\hline & & BW & $22.40 \pm 4.03$ & & $6.94 \pm 5.62$ & & $4.45 \pm 4.45$ & \\
\hline & \multirow{2}{*}{$0 \%$} & FW & $-2.41 \pm 3.67$ & \multirow{2}{*}{$0.001^{*}$} & $3.95 \pm 3.90$ & \multirow{2}{*}{$0.001^{*}$} & $17.45 \pm 3.28$ & \multirow{2}{*}{$0.001^{*}$} \\
\hline & & BW & $25.42 \pm 4.17$ & & $3.95 \pm 3.90$ & & $17.45 \pm 3.48$ & \\
\hline & \multirow{2}{*}{$+5 \%$} & FW & $0.91 \pm 4.35$ & \multirow{2}{*}{$0.001^{*}$} & $4.51 \pm 4.33$ & \multirow{2}{*}{$0.001^{*}$} & $15.74 \pm 3.16$ & \multirow{2}{*}{$0.001^{*}$} \\
\hline & & BW & $32.01 \pm 4.12$ & & $13.12 \pm 5.90$ & & $1.22 \pm 3.31$ & \\
\hline & \multirow{2}{*}{$+10 \%$} & FW & $8.06 \pm 5.39$ & \multirow{2}{*}{$0.001^{*}$} & $5.88 \pm 4.20$ & ก0०1* & $13.67 \pm 2.90$ & 0 \\
\hline & & BW & $35.29 \pm 4.35$ & & $17.55 \pm 6.51$ & 0.001 & $2.31 \pm 4.07$ & 0.001 \\
\hline & $-7.5 \%$ & FW & $8.62 \pm 4.12$ & ०012* & $-0.48 \pm 4.19$ & 0088 & $7.67 \pm 4.31$ & 0,273 \\
\hline & 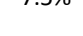 & BW & $4.95 \pm 3.77$ & 0.012 & $-2.15 \pm 3.24$ & 0.000 & $5.98 \pm 4.60$ & 0.275 \\
\hline & $0 \%$ & FW & $8.12 \pm 4.11$ & $0.005 *$ & $-0.77 \pm 4.58$ & ० १९९ & $11.35 \pm 5.15$ & 0.079 \\
\hline Ankle & & BW & $4.10 \pm 3.48$ & & $-0.77 \pm 3.91$ & & $8.59 \pm 6.95$ & \\
\hline (angle) & $5 \%$ & FW & $7.84 \pm 3.50$ & ค०००1* & $-2.51 \pm 4.21$ & 0010* & $12.97 \pm 4.78$ & $0002 *$ \\
\hline & $10 \%$ & BW & $2.84 \pm 3.53$ & 0.001 & $0.04 \pm 3.69$ & 0.010 & $7.80 \pm 5.76$ & 0.003 \\
\hline & $+10 \%$ & FW & $5.63 \pm 3.97$ & ค०००1* & $-5.12 \pm 4.49$ & ก001* & $15.10 \pm 5.79$ & (972 \\
\hline & & BW & $1.67 \pm 3.31$ & & 2.77 & & $15.18 \pm 8.86$ & \\
\hline
\end{tabular}

* Indicates a significant difference between FW and BW $(\mathrm{P}<0.05)$

PHYSICAL TREA MENTS

Positive values for the hip and knee represent the flexion and the negative values indicate extension. Positive values for ankle joint indicate plantar flexion and negative values indicate dorsiflexion.

the joint and as a result, increasing the risk of osteoarthri- inclines decreases knee adduction [9]. By increasing the tis in younger ages $[24,25]$.

Regarding FW, findings of this research, in line with Haggerty et al. (2014) study results showed that upward incline of the walking, knee joint has more flexion at the moment of heel contact compared to the normal walking. On the contrary, when walking downward inclines, the knee joint at the moment of the heel contact has more

Table 4. Knee angle variable presented as mean $\pm S D$ on the frontal plane during walking forward and backward on different inclines

\begin{tabular}{|c|c|c|c|c|c|c|c|c|}
\hline Joint & Incline & FW/BW & $\begin{array}{l}\text { Heel Contact Moment in } \\
\text { FW and Toe Contact in BW }\end{array}$ & $\mathbf{P}$ & Mid-Stance & $\mathbf{P}$ & $\begin{array}{l}\text { Heel-off Moment in FW } \\
\text { and Toe-off in BW }\end{array}$ & $\mathbf{P}$ \\
\hline \multirow{8}{*}{$\begin{array}{l}\text { Knee } \\
\text { (angle) }\end{array}$} & \multirow{2}{*}{$-7.5 \%$} & FW & $1.28 \pm 1.68$ & \multirow{2}{*}{$0.0018 *$} & $0.40 \pm 1.53$ & \multirow{2}{*}{0.134} & $3.10 \pm 2.13$ & \multirow{2}{*}{$0.001 *$} \\
\hline & & BW & $-0.19 \pm 1.91$ & & $-0.07 \pm 1.17$ & & $1.14 \pm 1.56 \pm$ & \\
\hline & \multirow{2}{*}{$0 \%$} & FW & $0.92 \pm 1.39$ & \multirow{2}{*}{0.080} & $0.38 \pm 1.62$ & \multirow{2}{*}{0.525} & $1.57 \pm 1.61$ & \multirow{2}{*}{0.110} \\
\hline & & BW & $-0.11 \pm 2.36$ & & $0.17 \pm 1.48$ & & $1.02 \pm 1.51$ & \\
\hline & \multirow{2}{*}{$+5 \%$} & $\mathrm{FW}$ & $0.68 \pm 1.44$ & \multirow{2}{*}{0.494} & $0.14 \pm 1.46$ & \multirow{2}{*}{0.914} & $0.83 \pm 1.56$ & \multirow{2}{*}{0.896} \\
\hline & & BW & $0.22 \pm 2.61$ & & $0.18 \pm 1.29$ & & $0.79 \pm 1.44$ & \\
\hline & \multirow{2}{*}{$+10 \%$} & FW & $0.27 \pm 1.57$ & \multirow{2}{*}{0.399} & $-0.13 \pm 1.50$ & \multirow{2}{*}{$0.004 *$} & $0.64 \pm 1.27$ & \multirow{2}{*}{0.289} \\
\hline & & BW & $0.79 \pm 2.40$ & & $0.90 \pm 1.60$ & & $0.89 \pm 1.37$ & \\
\hline
\end{tabular}

* Indicates a significant difference between FW and BW $(\mathrm{P}<0.05)$

PHYSICAL TREA MENTS

Positive values for knee joint indicate adduction and negative values indicate abduction. 
extension than walking without inclines [26]. As a result, knee joint is in close-packed position when walking down the inclines. In this situation, the ability to absorb joint force decreases and consequently, at the moment of heel contact where the initial peak of GFR exerted to the individual [21], the highest amount of knee adduction is seen compared to other inclines. As the incline degree increases, the walking mechanism changes, the joints get closer to the loose-packed position, the ability to absorb joint force increases, and as a result, the amount of adduction reduces and accordingly the torque adduction decreases. Therefore, the least adduction angle was seen during walking on an incline of $+10 \%$. However in $\mathrm{BW}$, the results were the opposite of FW and showed that the upward incline increases knee joint adduction angle and the downward incline reduces the abduction and even at moments, adduction is turned into abduction. This knee position change from adduction to abduction turns the knee adduction torque into abduction torque and finally reduces applied loads to the medial knee joint compartment.

Regarding the study limitations, we can mention subjects' different familiarity with walking on the treadmill. Installing reflective markers on subjects' bodies might also affect their walking. It is also suggested that kinetic parameters be investigated in future studies using tools such as electromyography and the force plate to complete the results of this research.

In sum, according to the findings of this research, it can be concluded that using FW exercises on the upward inclines or using BW exercises on the downward inclines may be an appropriate way to reduce the angle of knee adduction and, consequently, lower the load on the knee joint medial compartment.

\section{Acknowledgments}

The authors hereby express their sincere gratitude to all study subjects and other people who helped us with this research and also Shahid Bahonar University of Kerman that provided the opportunity to do our research.

\section{Conflict of Interest}

The authors declared no conflicts of interest.

\section{References}

[1] Chaloupka EC, Kang J, Mastrangelo MA, Donnelly MS. Cardiorespiratory and metabolic responses during forward and backward walking. Journal of Orthopaedic \& Sports Physical Therapy. 1997; 25(5):302-6. doi: 10.2519/jospt.1997.25.5.302

[2] Cipriani DJ, Armstrong CW, Gaul S. Backward walking at three levels of treadmill inclination: An electromyographic and kinematic analysis. Journal of Orthopaedic \& Sports Physical Therapy. 1995; 22(3):95-102. doi: 10.2519/ jospt.1995.22.3.95

[3] Hooper TL, Dunn DM, Props JE, Bruce BA, Sawyer SF, Daniel JA. The effects of graded forward and backward walking on heart rate and oxygen consumption. Journal of Orthopaedic \& Sports Physical Therapy. 2004; 34(2):65-71. doi: 10.2519/jospt.2004.34.2.65

[4] Terblanche E, Venter R. The effect of backward training on the speed, agility and power of netball players. South African Journal for Research in Sport, Physical Education and Recreation. 2009; 31(2):135-45. doi: 10.4314/sajrs.v31i2.46335

[5] Threlkeld AJ, Horn TS, Wojtowicz GM, Rooney JG, Shapiro R. Pilot study: Kinematics, ground reaction force, and muscle balance produced by backward running. Journal of Orthopaedic \& Sports Physical Therapy. 1989; 11(2):56-63. doi: 10.2519/jospt.1989.11.2.56

[6] Franz JR, Kram R. The effects of grade and speed on leg muscle activations during walking. Gait \& Posture. 2012; 35(1):143-7. doi: 10.1016/j.gaitpost.2011.08.025

[7] Silder A, Besier T, Delp SL. Predicting the metabolic cost of incline walking from muscle activity and walking mechanics. Journal of Biomechanics. 2012; 45(10):1842-9. doi: 10.1016/j.jbiomech.2012.03.032

[8] Ehlen KA, Reiser RFI, Browning RC. Energetics and biomechanics of inclined treadmill walking in obese adults. Medicine \& Science in Sports \& Exercise. 2011; 43(7):1251-9. doi: 10.1249/mss.0b013e3182098a6c

[9] Haggerty M, Dickin DC, Popp J, Wang H. The influence of incline walking on joint mechanics. Gait \& Posture. 2014; 39(4):1017-21. doi: 10.1016/j.gaitpost.2013.12.027

[10] Naderi S, Mohammadipour F, Amir Seyfaddini M. The effects of different walking inclinations on knee angle in the frontal plane of patients with varus malalignment. Physical Treatments-Specific Physical Therapy Journal. 2014; 4(3):139-44.

[11] Andriacchi TP. Dynamics of knee malalignment. The Orthopedic clinics of North America. 1994; 25(3):395-403. PMID: 8028883

[12] Hurwitz DE, Sumner DR, Andriacchi TP, Sugar DA. Dynamic knee loads during gait predict proximal tibial bone distribution. Journal of Biomechanics. 1998; 31(5):423-30. doi: 10.1016/s0021-9290(98)00028-1

[13] Sharma L. The role of knee alignment in disease progression and functional decline in knee osteoarthritis. JAMA 2001; 286(2):188. doi: 10.1001/jama.286.2.188

[14] Schipplein OD, Andriacchi TP. Interaction between active and passive knee stabilizers during level walking. Journal of Orthopaedic Research. 1991; 9(1):113-9. doi: 10.1002/ jor.1100090114 
[15] Faul F, Erdfelder E, Lang AG, Buchner A. G*Power 3: A flexible statistical power analysis program for the social, behavioral, and biomedical sciences. Behavior Research Methods. 2007; 39(2):175-91. doi: 10.3758/bf03193146

[16] Werner C, Lindquist AR, Bardeleben A, Hesse S. The influence of treadmill inclination on the gait of ambulatory hemiparetic subjects. Neurorehabilitation and Neural Repair. 2007; 21(1):76-80. doi: 10.1177/1545968306289958

[17] Munro A, Herrington L, Comfort P. Comparison of landing knee valgus angle between female basketball and football athletes: Possible implications for anterior cruciate ligament and patellofemoral joint injury rates. Physical Therapy in Sport. 2012; 13(4):259-64. doi: 10.1016/j.ptsp.2012.01.005

[18] Rodenbusch TLM, Ribeiro TS, Simão CR, Britto HMJS, Tudella E, Lindquist AR. Effects of treadmill inclination on the gait of children with down syndrome. Research in Developmental Disabilities. 2013; 34(7):2185-90. doi: 10.1016/j. ridd.2013.02.014

[19] Moreno CC, Mendes LA, Lindquist AR. Effects of treadmill inclination on the gait of individuals with chronic hemiparesis. Archives of Physical Medicine and Rehabilitation. 2011; 92(10):1675-80. doi: 10.1016/j.apmr.2011.05.016

[20] Tulchin K, Orendurff M, Karol L. The effects of surface slope on multi-segment foot kinematics in healthy adults. Gait \& Posture. 2010; 32(4):446-50. doi: 10.1016/j.gaitpost.2010.06.008

[21] Chung MJ, Wang MJJ. The change of gait parameters during walking at different percentage of preferred walking speed for healthy adults aged 20-60 years. Gait \& Posture. 2010; 31(1):131-5. doi: 10.1016/j.gaitpost.2009.09.013

[22] Zeni JA, Richards JG, Higginson JS. Two simple methods for determining gait events during treadmill and overground walking using kinematic data. Gait \& Posture. 2008; 27(4):710-4. doi: 10.1016/j.gaitpost.2007.07.007

[23] Lee M, Kim J, Son J, Kim Y. Kinematic and kinetic analysis during forward and backward walking. Gait \& Posture. 2013; 38(4):674-8. doi: 10.1016/j.gaitpost.2013.02.014

[24] Tetsworth K, Paley D. Malalignment and degenerative arthropathy. Orthopedic Clinics of North America. 1994; 25(3):367-77. PMID: 8028880

[25] Brouwer GM, Tol AWV, Bergink AP, Belo JN, Bernsen RMD, Reijman M, et al. Association between valgus and varus alignment and the development and progression of radiographic osteoarthritis of the knee. Arthritis \& Rheumatism. 2007; 56(4):1204-11. doi: 10.1002/art.22515

[26] Han JT, Kwon YH, Park JW, Koo HM, Nam KS. Three-dimensional kinematic analysis during upslope walking with different inclinations by healthy adults. Journal of Physical Therapy Science. 2009; 21(4):385-91. doi: 10.1589/jpts.21.385 